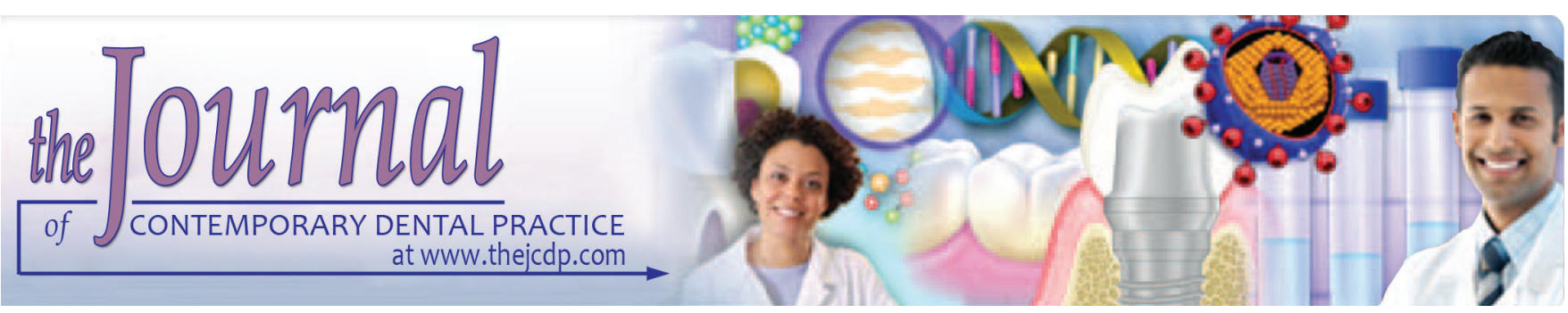

\title{
Assessment of Interexaminer Agreement in the Detection of Condyle Morphology and positioning with Two Methods: Radiographic and Tomographic
}

\author{
1 JS de Almeida Giunco, ${ }^{2} \mathrm{PV}$ Pedron Oltramari-Navarro, ${ }^{3} \mathrm{TM}$ Freire Fernandes \\ ${ }^{4} \mathrm{R}$ de Lima Navarro, ${ }^{5} \mathrm{MR}$ de Almeida, ${ }^{6} \mathrm{AC}$ de Castro Ferreira Conti
}

\begin{abstract}
Aim: This study aims at evaluating the interexaminer agreement between radiographic and tomographic methods to determine condyle morphological variations and positioning.

Materials and methods: The sample comprised 100 individuals aged 13 to 30 years, from the patient files of University of North Paraná. The assessment of condyles morphology and positioning was performed in images of digital panoramic radiography (DPR) and reconstructed panoramic images from the cone beam computed tomography (CBCT) scans, by using the Dolphin three-dimensional (3D) program. The condyle morphology was categorized as flat, convex, and angular as well as its positioning classified into anterior, posterior, and concentric. Three calibrated examiners performed this subjective evaluation. After that, another examiner performed an objective assessment of the condyles positioning using tomographic sagittal scans of the condyles, applying the same 3D program. This objective evaluation of the condyle position, considered the gold standard (GS), was achieved by using a formula based on the measurement values of the joint spaces, anterior and posterior. The kappa test was used to assess the interexaminer agreement in determining the condyles morphology and positioning, as well as between the condyle positioning results determined by the examiners and the GS.
\end{abstract}

Results: The results showed poor agreement among examiners and between the subjective and objective condyle positioning evaluation.

\footnotetext{
${ }^{1-3,5}$ Department of Orthodontics, University of North Parana Londrina, Paraná, Brazil

${ }^{4}$ Department of Dentistry, Area of Oral and Maxillofacial Surgery State University of Maringá, Maringá, Paraná, Brazil

${ }^{6}$ Department of Orthodontics, Sagrado Coração University Bauru, São Paulo, Brazil

Corresponding Author: AC de Castro Ferreira Conti Department of Orthodontics, Sagrado Coração University Bauru, São Paulo, Brazil, Phone: +5514997957795 , e-mail: accfconti@uol.com.br
}

Conclusion: It was concluded that the panoramic radiography $(\mathrm{PR})$, either digitalized or reconstructed from CBCT scans, is not suitable for determining variations in condyle morphology and position.

Clinical significance: Whenever it is necessary to evaluate the mandibular condyle during the orthodontic screening, the orthodontist should consider another image modality better than the PR.

Keywords: Cone beam computed tomography, Mandibular condyle, Panoramic radiography.

How to cite this article: de Almeida Giunco JS, OltramariNavarro PVP, Freire Fernandes TMF, de Lima Navarro R, de Almeida MR, de Castro Ferreira Conti AC. Assessment of Interexaminer Agreement in the Detection of Condyle Morphology and positioning with Two Methods: Radiographic and Tomographic. J Contemp Dent Pract 2016;17(10):837-843.

\section{Source of support: Nil}

Conflict of interest: None

\section{INTRODUCTION}

Attempts should be made to diagnose bone disorders in the temporomandibular joint (TMJ) because these conditions may be representative of signs and symptoms of temporomandibular dysfunction (TMD) or of changes in occlusion or in the position of the mandibular condyles. ${ }^{1,2}$

In this context, several imaging techniques have been described for assessing the TMJ. Panoramic radiographic images, which are frequently used for orthodontic diagnoses, are often used to assess the TMJ because this technique is widely available, easy to use, and involve low radiation and costs. ${ }^{3}$ Although many researchers use panoramic radiography (PR) to evaluate the changes in the condyles and the anatomical differences inherent to this joint, some factors, such as overlapping anatomical structures, X-ray projection angle, anatomical variations, 
and the position of the patient's head can compromise this evaluation. ${ }^{4-6}$

Imaging methods for evaluating the TMJ include transcranial radiography (TR), which is indicated for the diagnosis of mandibular condyle movement restriction in the presence of muscle spasm and pain, and posteroanterior and submentovertical X-rays, which identify condylar hyperplasia. ${ }^{7}$

The diagnostic accuracy of magnetic resonance image (MRI) and computed tomography (CT) is superior to those of conventional radiological examinations because of their higher resolution. Computed tomography is the ideal method for assessing bone structures, whereas MRI allows the study of soft parts, including the intra-articular disk. These two methods are often combined to study the TMJ disorders, and they are important tools in the differential diagnosis of diseases that affect this region. ${ }^{8}$

A study that compared the results of the position of the mandibular condyle obtained by TR and linear tomography reported that the results were more precise with tomographic images. Indeed, it was questioned whether $\mathrm{TR}$ is an appropriate method for measuring the position of the mandibular condyle. ${ }^{9}$

Although CT provides better visualization of the TMJ structures, the radiation dose is higher than radiography or linear tomography. The balance between the risks of radiation overdose and the benefits of the diagnostic superiority of these images have been discussed previously. ${ }^{10}$

After its development in the late 1990s, cone beam computed tomography (CBCT) has been widely used in dentistry, including orthodontics. The radiation dose used in CBCT is lower than that used in conventional medical tomography; thus, it is indicated for treatment planning and diagnoses in dentistry. The precision of this technique in detecting TMJ bone changes has been reported. ${ }^{4}$

Although efforts have been made to establish criteria for requesting CBCT images, many doubts remain regarding these criteria, especially those concerning the evaluation of the morphology and position of the mandibular condyle in the TMJ. The reliability of two-dimensional (2D) images for this purpose still needs to be clarified, especially with regard to panoramic X-rays, which are commonly requested as a component of orthodontic records and based on that radiographic reports are issued regarding the findings of TMJ structures. The validity of these information should be questioned, is this radiographic method suitable to detect TMJ bone changes?

Therefore, this study aimed to investigate the interexaminer agreement in evaluating the morphology and positioning of the condyle in PR images, either digitalized or reconstructed from CBCT scans.

\section{MATERIALS AND METHODS}

\section{Sample}

This study was approved by the Research Ethics Committee of the University of North Paraná, protocol number 276.661. Patients and patients' parents/guardians signed a consent form allowing the use of the orthodontic records in this study.

For the sample selection, 180 patient records from the orthodontic clinic were examined according to the following inclusion criteria: Patients between 13 and 30 years of age without previous orthodontic treatment, with complete permanent dentition (with the exception of the third molars), without a history of TMD treatment. After applying these criteria, 101 records composed of panoramic X-rays and CBCT scans were included in this study.

\section{Methods}

The panoramic X-rays were obtained by using an ortho pantomograph OP 100 (Instrumentarium Corp., Tuusula, Finland) panoramic $X$-ray machine, with a magnification index of $30 \%$. The following protocol was used: 17.6 seconds, $57 \mathrm{KVp}$, and $2 \mathrm{~mA}$. The patients' heads were aligned with the Frankfort plane, parallel to the ground, and an acrylic device was placed between the anterior teeth.

The CBCT images were obtained by using tomographer (i-CAT; Imaging Sciences, $\mathrm{KaVo}^{\circledR}$, Hatfield, PA, USA), with a standardized head position. The following specifications were used: $2216 \mathrm{~cm}$ field of view (FOV), 40 seconds of exposure, $120 \mathrm{KVp}$, and $36 \mathrm{~mA}$. This machine has a high-resolution sensor that allows images to be obtained with a voxel (volumetric picture element) of $0.4 \mathrm{~mm}$. During panoramic X-ray reconstruction, the arch dimensions (thickness and width) were adjusted according to the size of the patients' maxilla.

In order to perform the subjective evaluation of condyle morphology and position, three previously calibrated examiners analyzed the right and left TMJs images of the digital panoramic X-rays (Fig. 1) and the CBCT-reconstructed panoramic X-rays (Fig. 2). Examiner calibration was performed using 10 images of each method, in which all experienced in TMJ evaluation examiners (one orthodontist, one radiologist, and one oral surgeon), openly classified the condyle morphology and position, and any questions regarding the different classification were discussed. The condyle morphology was categorized as flat, convex, and angular. In addition, criteria, such as the presence of asymmetries, bifid mandibular condyle, or the presence of osteophytes were considered. The position of the condyle in relation to the mandibular fossa was categorized as anterior, posterior, 


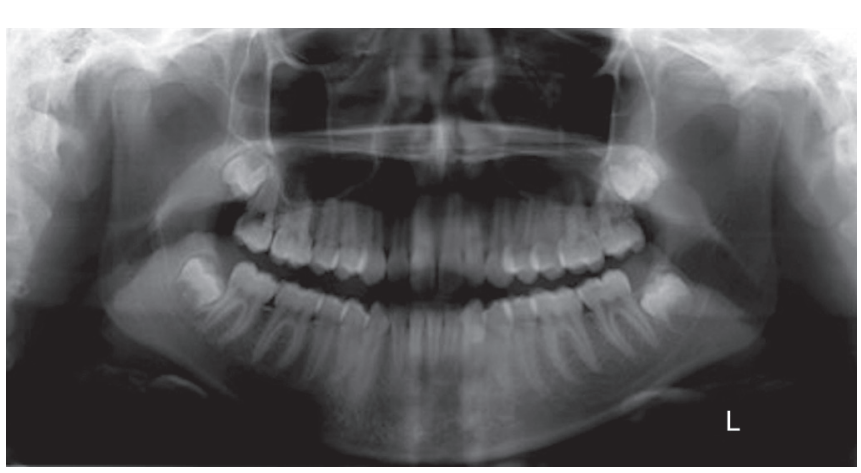

Fig. 1: Digital panoramic X-rays

and concentric. This evaluation was performed by using Dolphin Imaging $11.5^{\mathrm{R}}$ computer software (Dolphin Imaging and Management Solutions, Chatsworth, CA, USA). The examiners performed this interpretation in a dark room, without interferences. They were asked to analyze all panoramic X-rays and then answer a questionnaire about the position and morphology of the mandibular condyles.

Another calibrated examiner performed the objective evaluation of the mandibular condyle position, considered as the gold standard (GS). The same program (Dolphin $11.5^{\mathrm{R}}$ ) was used to determine the condyle positioning by using the sagittal sections of the CBCT images of the right and left TMJs. An image acquisition protocol was developed to obtain standardized sections. Initially, the head position was standardized. The horizontal plane was aligned with the orbits in a vertical plane passing through the glabella and nasal septum in the frontal view. The skull was repositioned relative to the Frankfort horizontal plane. Subsequently, a sagittal reconstruction of the TMJ was obtained (Fig. 3) to determine the area of the axial sections with the greatest distance between the poles of the mandibular condyle on each side. In this position, the most central point of the mandibular condyle was marked (Fig. 4).

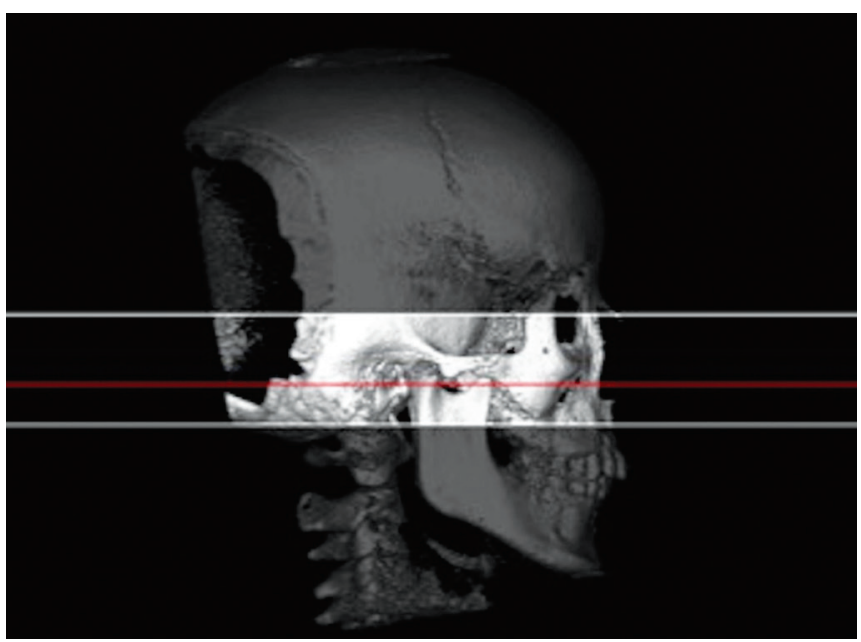

Fig. 3: Sagittal view of the TMJ region to obtain the axial reconstruction

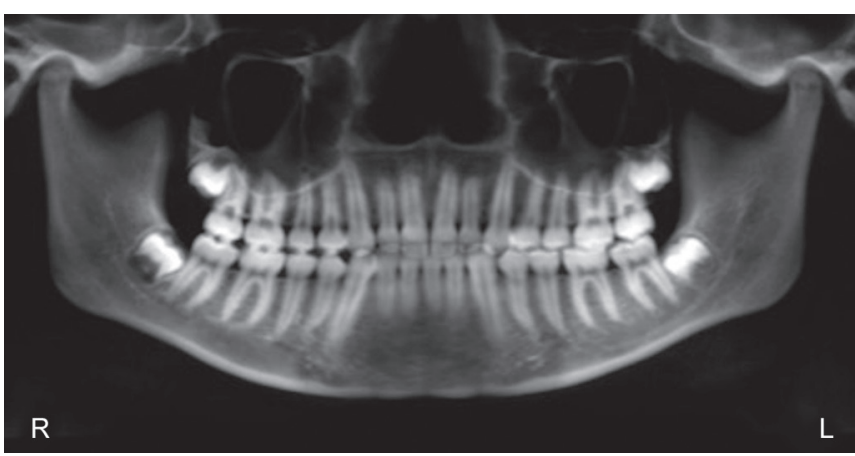

Fig. 2: Panoramic X-rays-reconstructed from $\mathrm{CBCT}$

The anterior and posterior articular spaces were measured in the most central sagittal section, on both sides. The condylar concentricity was measured based on the formula proposed by Pullinger and Hollender, ${ }^{11}$ as shown below.

$$
\frac{P-A}{P+A} \times 100(\%)
$$

The narrowest anterior $(\mathrm{A})$ and posterior $(\mathrm{P})$ articular spaces were calculated by using linear measurements in the right and left TMJs (Figs 5A and B). The concentric condyle in the articular fossa was indicated by a zero result, whereas a negative value indicated a posterior location and a positive value indicated an anterior location. This classification of condyle position GS was subsequently compared with the results of the subjective analyses performed by the three examiners.

\section{Statistical Analysis}

The kappa test was used to assess interexaminer agreement on the positioning and morphological changes of the mandibular condyle assessed by the two methods, i.e., panoramic X-rays and CBCT-reconstructed panoramic X-rays. The kappa test was also used to evaluate

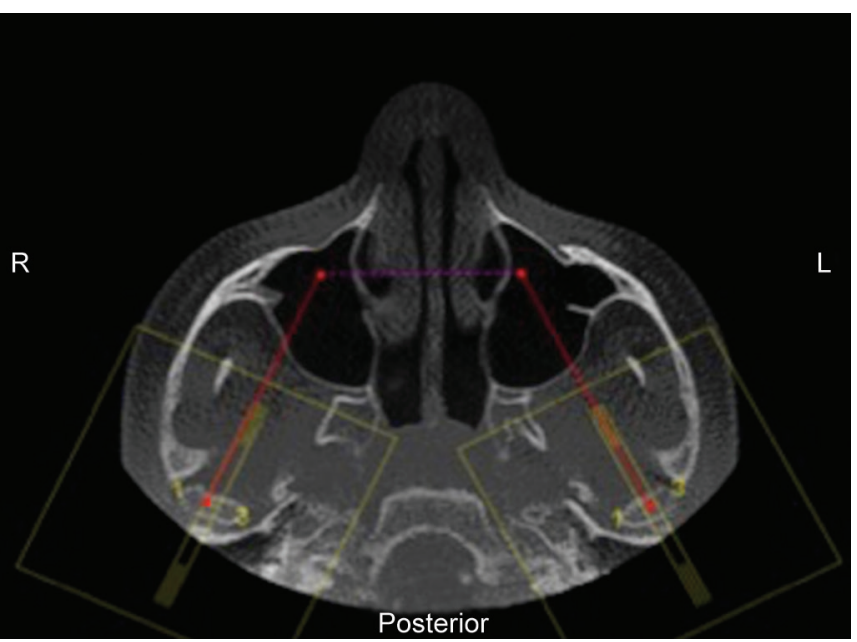

Fig. 4: Axial images of the TMJ 

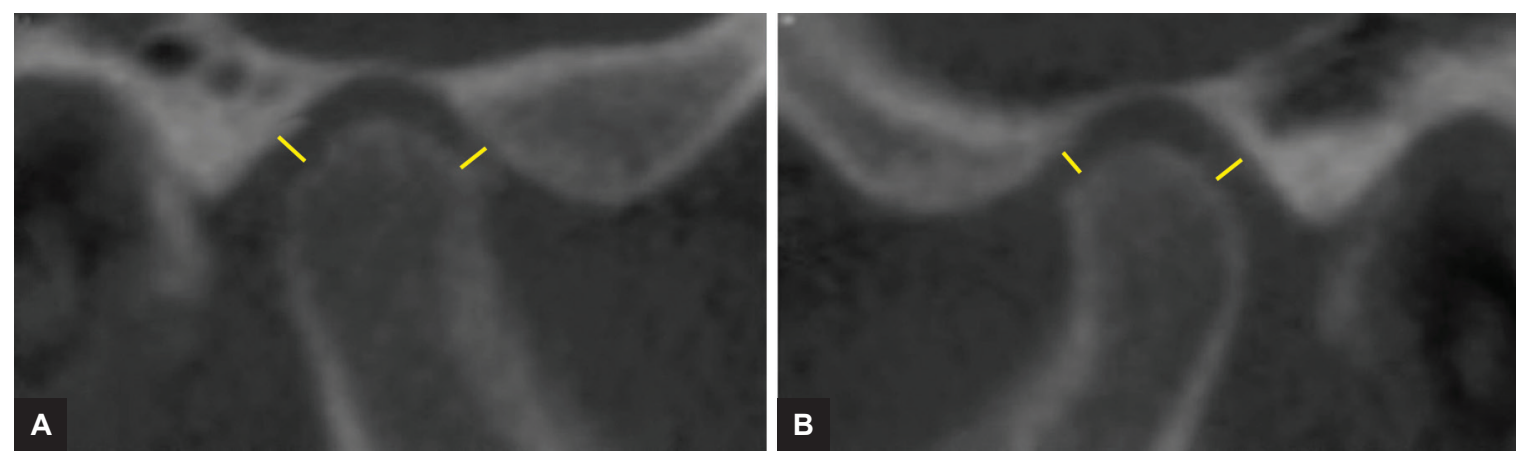

Figs 5A and B: Smallest anterior and posterior articular spaces: (A) Right TMJ; and (B) left TMJ

Table 1: Condyle position (right and left TMJ) between examiners. Evaluation of the DPR and the GS

\begin{tabular}{|c|c|c|c|c|c|c|c|c|}
\hline \multirow[b]{2}{*}{ Condyle position } & \multicolumn{2}{|c|}{ Examiner 1} & \multicolumn{2}{|c|}{ Examiner 2} & \multicolumn{2}{|c|}{ Examiner 3} & \multicolumn{2}{|c|}{ Gold standard } \\
\hline & Right TMJ & Left TMJ & Right TMJ & Left TMJ & Right TMJ & Left TMJ & Right TMJ & Left TMJ \\
\hline Concentric & 58 & 53 & 19 & 19 & 1 & 2 & 15 & 8 \\
\hline Anterior & 30 & 37 & 81 & 82 & 97 & 97 & 55 & 61 \\
\hline Posterior & 101 & 101 & 1 & 0 & 3 & 2 & 31 & 32 \\
\hline Total & 101 & 101 & 101 & 101 & 101 & 101 & 101 & 101 \\
\hline
\end{tabular}

Table 2: Condyle position (right and left TMJ) between examiners. Evaluation of the PR reconstructed from CBCT and the GS

\begin{tabular}{|c|c|c|c|c|c|c|c|c|}
\hline \multirow[b]{2}{*}{$\begin{array}{l}\text { Condyle } \\
\text { position }\end{array}$} & \multicolumn{2}{|c|}{ Examiner 1} & \multicolumn{2}{|c|}{ Examiner 2} & \multicolumn{2}{|c|}{ Examiner 3} & \multicolumn{2}{|c|}{$\begin{array}{c}\text { Gold } \\
\text { standard }\end{array}$} \\
\hline & $\begin{array}{l}\text { Right } \\
\text { TMJ }\end{array}$ & $\begin{array}{l}\text { Left } \\
\text { TMJ }\end{array}$ & $\begin{array}{l}\text { Right } \\
\text { TMJ }\end{array}$ & $\begin{array}{l}\text { Left } \\
\text { TMJ }\end{array}$ & $\begin{array}{l}\text { Righ } \\
\text { TMJ }\end{array}$ & $\begin{array}{l}\text { Left } \\
\text { TMJ }\end{array}$ & $\begin{array}{l}\text { Right } \\
\text { TMJ }\end{array}$ & $\begin{array}{l}\text { Left } \\
\text { TMJ }\end{array}$ \\
\hline Concentric & 62 & 57 & 46 & 47 & 22 & 26 & 15 & 8 \\
\hline Anterior & 29 & 35 & 41 & 41 & 56 & 49 & 55 & 61 \\
\hline Posterior & 10 & 9 & 14 & 13 & 23 & 26 & 31 & 32 \\
\hline Total & 101 & 101 & 101 & 101 & 101 & 101 & 101 & 101 \\
\hline
\end{tabular}

Table 4: Condyle morphology (right and left TMJ) between examiners. Evaluation of the PR reconstructed from CBCT scans

\begin{tabular}{|c|c|c|c|c|c|c|}
\hline \multirow[b]{2}{*}{$\begin{array}{l}\text { Condyle } \\
\text { morphology }\end{array}$} & \multicolumn{2}{|c|}{ Examiner 1} & \multicolumn{2}{|c|}{ Examiner 2} & \multicolumn{2}{|c|}{ Examiner 3} \\
\hline & $\begin{array}{l}\text { Right } \\
\text { TMJ }\end{array}$ & $\begin{array}{l}\text { Left } \\
\text { TMJ }\end{array}$ & $\begin{array}{l}\text { Right } \\
\text { TMJ }\end{array}$ & $\begin{array}{l}\text { Left } \\
\text { TMJ }\end{array}$ & $\begin{array}{l}\text { Right } \\
\text { TMJ }\end{array}$ & $\begin{array}{l}\text { Left } \\
\text { TMJ }\end{array}$ \\
\hline Flat & 9 & 13 & 20 & 8 & 11 & 9 \\
\hline Convex & 82 & 78 & 76 & 82 & 88 & 90 \\
\hline Angular & 10 & 10 & 5 & 11 & 2 & 2 \\
\hline Total & 101 & 101 & 101 & 101 & 101 & 101 \\
\hline
\end{tabular}

the agreement between the results of the examiners and the GS with regard to the condyle position. The kappa values according to Laandis and Koch ${ }^{12}$ were used for its interpretation.

\section{RESULTS}

Regarding the condylar positioning, the evaluation performed on the digital PR revealed that examiner 1 found more concentric condyles in contrast with examiners 2 and 3 who found anteriorly displaced condyles in the most (Table 1). When the evaluation was performed in the PR reconstructed from CBCT scans, examiner 1 still
Table 3: Condyle morphology (right and left TMJ) between examiners. Evaluation of the DPR

\begin{tabular}{lllllllll}
\hline & \multicolumn{2}{c}{ Examiner 1 } & & \multicolumn{2}{c}{ Examiner 2 } & & \multicolumn{2}{c}{ Examiner 3 } \\
\cline { 2 - 3 } Condyle & Right & Left & & Right & Left & & Right & Left \\
position & TMJ & TMJ & & TMJ & TMJ & & TMJ & TMJ \\
\hline Flat & 0 & 1 & & 7 & 8 & & 3 & 2 \\
Convex & 97 & 94 & & 91 & 90 & & 95 & 95 \\
Angular & 4 & 6 & & 3 & 3 & 3 & 4 \\
\hline Total & 101 & 101 & 101 & 101 & & 101 & 101 \\
\hline
\end{tabular}

found more concentric condyles, examiner 2 found almost equally concentric and anteriorly displaced condyles, while examiner 3 found more anteriorly displaced condyle (Table 2). The GS results indicated the following: for the right condyles 55 anteriorly displaced, 15 concentric, and 31 posterior; for the left condyles, 61 were anterior, 8 concentric, and 32 posterior (Tables 1 and 2).

When considering the condylar morphology, the evaluation performed on the digital panoramic radiography (DPR) revealed that examiners 1,2 and 3 found the condyle convex shape in the most of the TMJ assessed (Table 3). These results are similar when the evaluation was performed in the PR reconstructed from CBCT scans (Table 4).

The agreement between the three examiners in the evaluation of the panoramic X-rays was very low, particularly with regard to the position of the condyle; in contrast, the agreement on symmetry and morphology was more significant (Table 5).

When CBCT-reconstructed panoramic images were applied, the agreement results were superior to those for digital radiography (Table 6). 
Assessment of Interexaminer Agreement in the Detection of Condyle Morphology and positioning with Two Methods

Table 5: Interexaminers agreement of the TMJ assessment in the DPR (kappa test)

\begin{tabular}{|c|c|c|c|c|c|c|}
\hline & \multicolumn{2}{|c|}{$E X 1 \times E X 2$} & \multicolumn{2}{|c|}{$E X 1 \times E X 3$} & \multicolumn{2}{|c|}{$E X 2 \times E X 3$} \\
\hline & \% concord. & карра & $\%$ concord. & kappa & \% concord. & kappa \\
\hline Right TMJ position & 40 & 0.08 & 32 & 0.03 & 79 & 0.05 \\
\hline Left TMJ position & 44 & 0.07 & 39 & 0.04 & 79 & 0.00 \\
\hline Simetry & 84 & 0.25 & 90 & 0.12 & 84 & 0.15 \\
\hline Right morphology & 73 & 0.25 & 82 & 0.38 & 79 & 0.35 \\
\hline Left morphology & 75 & 0.28 & 78 & 0.29 & 85 & 0.44 \\
\hline
\end{tabular}

Table 6: Interexaminers agreement of the TMJ assessment in the CBCT-reconstructed PR (kappa test)

\begin{tabular}{|c|c|c|c|c|c|c|}
\hline & \multicolumn{2}{|c|}{$E X 1 \times E X 2$} & \multicolumn{2}{|c|}{$E X 1 \times E X 3$} & \multicolumn{2}{|c|}{$E X 2 \times E X 3$} \\
\hline & \%concord. & kappa & \%concord. & kappa & \%concord. & kappa \\
\hline Right TMJ position & 64 & 0.39 & 56 & 0.36 & 63 & 0.42 \\
\hline Left TMJ position & 68 & 0.46 & 59 & 0.38 & 57 & 0.34 \\
\hline Simetry & 79 & 0.04 & 86 & 0.06 & 83 & 0.03 \\
\hline Right morphology & 88 & 0.17 & 92 & 0.17 & 85 & 0.06 \\
\hline Left morphology & 85 & 0.12 & 93 & 0.43 & 87 & 0.19 \\
\hline
\end{tabular}

Table 7: Agreement between the objective TMJ positioning evaluation, GS, and the TMJ assessment in DPR and CBCTreconstructed panoramic radiography $(\mathrm{RPR})$, kappa test

\begin{tabular}{|c|c|c|c|c|c|}
\hline \multirow{2}{*}{ Examiner } & & \multicolumn{2}{|c|}{$D P R \times G S$} & \multicolumn{2}{|c|}{$R P R \times G S$} \\
\hline & & \%concord. & kappa & \%concord. & kappa \\
\hline \multirow[t]{2}{*}{ EX1 } & Right TMJ & 35 & 0.09 & 38 & 0.14 \\
\hline & Left TMJ & 35 & 0.07 & 40 & 0.16 \\
\hline \multirow[t]{2}{*}{ EX2 } & Right TMJ & 50 & 0.06 & 50 & 0.25 \\
\hline & Left TMJ & 51 & 0.01 & 48 & 0.23 \\
\hline \multirow[t]{2}{*}{ EX3 } & Right TMJ & 56 & 0.07 & 55 & 0.25 \\
\hline & Left TMJ & 61 & 0.06 & 53 & 0.22 \\
\hline \multirow{2}{*}{$\begin{array}{l}\text { All } \\
\text { examiners }\end{array}$} & Right TMJ & 52 & 0.07 & 46 & 0.17 \\
\hline & Left TMJ & 58 & 0.08 & 51 & 0.26 \\
\hline
\end{tabular}

The agreement on the position of the mandibular condyle between the subjective analyses and the GS was also very low (Table 7); the results of the CBCTreconstructed panoramic images were better for examiners 2 and 3 .

\section{DISCUSSION}

Discerning the characteristics of the TMJ may have important clinical implications for the orthodontic diagnosis, prognosis, and treatment plan..$^{13}$ Therefore, when necessary, the clinician should request the imaging technique that is most appropriate for each patient, while considering the advantages and disadvantages of each method. ${ }^{14}$

In this context, the orthodontist often receives reports based on PR images that infer the position and the morphological findings of the mandibular condyles. The validity of these images for determining changes in the TMJ should be considered, and this was the objective of this study.

The results showed that the condyle positioning varied between examiners, while examiner 1 found most condyles concentric, examiners 2 and 3 found more anteriorly displaced condyles in the DPRs (Table 1). When the images were reconstructed from CBCT scans, the results indicated more concentric condyles (Table 2), which was expected as the PR examinations are taken asking the patient to bite an occlusal device that could displace the mandible forward.

Condyle morphology results indicated that the majority of the condyles presented a convex shape, regardless the exam protocol, PRs or PRs reconstructed from CBCT scans (Tables 3 and 4 ).

Taking into account the condyle position assessed in the PRs, the most important finding is that no significant agreement was observed between the examiners (Table 5). With regard to morphology and the presence of asymmetries, the percentage of agreement was high despite the low kappa values; this was probably due to a high prevalence of convex condyle and a low prevalence of asymmetries, which was expected in a young population. However, asymmetries should be evaluated with care in PR. ${ }^{15}$

Even when the panoramic $X$-rays were reconstructed from $\mathrm{CBCT}$ scans, which presumably improves image quality, a low interexaminer agreement on the position of the condyles was observed according to the kappa index (Table 6). The reason to create a panoramic view from CBTC was just to allow drawing conclusions after comparing two multiplanar images. As can be noticed in the results, none of them achieved acceptable values of agreement. This fact strengthens the need for regular sagittal images when condyle positioning is studied. However, the agreement among these results was better than were those of digital radiography. With regard to morphology and the presence of asymmetries, the kappa values were also low and the percentage of interexaminer agreement was high. 
According to the results of this study, PR images are not indicated for the evaluation of the position and morphology of the TMJ because the findings cannot be reproduced. Panoramic radiography is indicated for an ample view of the maxilla and mandible. ${ }^{16,17}$ However, this imaging method is not indicated for the inspection and functional interpretation of the TMJ because the patient is instructed to bite on an acrylic device during the examination, causing advancement of the mandible and the inferior teeth. This changes the mandibular condyle-fossa relationship, hindering the evaluation. ${ }^{18}$ Moreover, because it is a transfacial exposure, the lateral pole of the condyle projects onto itself, limiting the image of the median portion; thus, surrounding structures may overlap the normal mandible components, simulating disorders that do not exist. ${ }^{19}$ Indeed, the influence of head positioning during image acquisition also accounts for this limitation, ${ }^{20}$ as well as the image formation, as the condyles are angled $\left(15-33^{\circ}\right)$ to sagittal plane making the X-ray beam projection obliquely to the condyle axis. ${ }^{21}$

Tomographic scan is currently the method of choice in the evaluation of joint erosion or osteophytes. ${ }^{22}$ Three independent observers compared $\mathrm{CBCT}$ and $\mathrm{CT}$ images of the TMJ and analyzed the changes in the mandibular condyle (flattening, defects, and osteophytes) and in the articular tubercle (flattening and defects). No significant difference was found between the two imaging methods; a significant difference was found only in the lateral view. ${ }^{23}$ Therefore, CT and CBCT are the methods of choice to evaluate changes in the mandibular condyle; however, considering the difference in radiation dose, CBCT is probably the more conservative option.

Furthermore, the present study showed a low agreement between the evaluations of the three examiners and the results of the GS (Table 7). This confirms that PR is not valid for determining the position of the condyle. These results highlight the need to request $\mathrm{CBCT}$ images when an evaluation of the morphology and positioning of the TMJ structures is necessary. Moreover, controversy exists regarding the condyle position inside the mandibular fossa, ${ }^{24,25}$ and no quantitative patterns are available for the ideal position of the condyle. ${ }^{26}$ Although one could think that bilateral symmetry of the condyles are necessary for absence of clinical symptoms of TMD, an association between concentric condyle-fossa relationships and abnormal TMJ function has been reported. ${ }^{11}$

The present study confirms that determining the condyle position and its morphology based on PR imaging is a very complex task. Other than ruling out significant alteration in bone, such as fracture and advanced changes of shape, the use of PR may not add to the diagnosis or clinical management of TMD patients. In such cases, clinical findings may indicate the diagnosis or need for detailed imaging of joint structures. ${ }^{5}$ When additional information is needed, other imaging methods can be required to obtain a more reliable diagnosis.

\section{CONCLUSION}

Based on the poor interexaminer agreement found in this study, PR, whether it is digital or CBCT-reconstructed, may not be suitable for evaluating the morphology and the position of the mandibular condyle.

\section{CLINICAL SIGNIFICANCE}

Whenever it is necessary to evaluate the mandibular condyle during the orthodontic screening, the orthodontist should consider another image modality better than the PR, even though this last one is always requested for the orthodontic diagnosis.

\section{REFERENCES}

1. Akerman S. Morphologic, radiologic and thermometric assessment of degenerative and inflammatory temporomandibular joint disease. An autopsy and clinical study. Swed Dent J Suppl 1987;52:1-110.

2. Tsuruta A, Yamada K, Hanada K, Koyama JI, Hayashi T, Hosogai A, Kohno S. Comparison of condylar positions at intercuspal and reference positions in patients with condylar bone change. J Oral Rehabil 2004 Jul;31(7):640-646.

3. Brooks SL, Brand JW, Gibbs SJ, Hollender L, Lurie AG, Omnell KA, Westesson PL, White SC. Imaging of the temporomandibular joint: a position paper of the American Academy of Oral and Maxillofacial Radiology. Oral Surg Oral Med Oral Pathol Oral Radiol Endod 1997 May;83(5): 609-618.

4. Hilgers ML, Scarfe WC, Scheetz JP, Farman AG. Accuracy of linear temporomandibular joint measurements with cone beam computed tomography and digital cephalometric radiography. Am J Orthod Dentofacial Orthop 2005 Dec;128(6):803-811.

5. Epstein JB, Caldwell J, Black G. The utility of panoramic imaging of the temporomandibular joint in patients with temporomandibular disorders. Oral Surg Oral Med Oral Pathol Oral Radiol Endod 2001 Aug;92(2):236-239.

6. Ludlow JB, Davies KL, Tyndall DA. Temporomandibular joint imaging: a comparative study of diagnostic accuracy for the detection of bone change with biplanar multidirectional tomography and panoramic images. Oral Surg Oral Med Oral Pathol Oral Radiol Endod 1995 Dec;80(6):735-743.

7. Smith NJ, Harris M. Radiology of the temporomandibular joint and condylar head. Br Dent J 1970 Oct;129(8):361-368.

8. Garcia MM, Machado KFS, Mascarenhas MH. Ressonância magnética e tomografia computadorizada da articulação temporomandibular: além da disfunção. Radiol Bras 2008 Sep-Oct;41(5):337-342.

9. Pullinger AG, Hollender L, Solberg WK, Petersson A. A tomographic study of mandibular condyle position in an asymptomatic population. J Prosthet Dent 1985 May;53(5):706-713.

10. Tsiklakis K, Donta C, Gavala S, Karayianni K, Kamenopoulou V, Hourdakis CJ. Dose reduction in maxillofacial imaging 
using low dose Cone Beam CT. Eur J Radiol 2005 Dec;56(3): 413-417.

11. Pullinger A, Hollender L. Variation condyle-fossa relationships according to different methods of evaluation in tomograms. Oral Surg Oral Med Oral Pathol 1986 Dec;62(6):719-727.

12. Landis JR, Koch GG. The measurement of observer agreement for categorical data. Biometrics 1977 Mar;33(1):159-174.

13. Katsavrias EG, Halazonetis DJ. Condyle and fossa shape in Class II and Class III skeletal patterns: a morphometric tomographic study. Am J Orthod Dentofacial Orthop 2005 Sep;128(3):337-346.

14. Moraes L, Duarte M, Medice Filho E, Moraes M. Imagens da ATM - Técnicas de exame. J Bras Ortodon Ortop Facial 2001;6(36):502-507.

15. Van Eslande DC, Russett SJ, Major PW, Flores-Mir C. Mandibular asymmetry diagnosis with panoramic imaging. Am J Orthod Dentofacial Orthop 2008 Aug;134(2):183-192.

16. Manzione JV. Radiographic evaluation. In: Ogus D, Toller A, editors. Common disorders of temporomandibular joint. 2nd ed. Bristol: John Wright, 1986. p. 40-62.

17. Payne M, Nakielny RA. Temporomandibular joint imaging. Clin Radiol 1996 Jan;51(1):1-10.

18. Pasler FA. Panoramic fadiography fo basic information and supplemental examination using special radiographys. In: Color atlas of dental medicine. Radiology. New York, NY: Thieme; 1993. p. 9-124.

19. Cristiansen EL, Thompson JR. Radiographic evaluation of the TMJ. In: Clinical management of temporomandibular disorders and orofacial pain. 1995. p. 161-174.
20. Markic G, Müller L, Patcas R, Roos M, Lochbühler N, Peltomäki T, Karlo CA, Ullrich O, Kellenberger CJ. Assessing the length of the mandibular ramusand the condylar process: a comparison of OPG, CBCT, CT, MRI, and lateral cephalometric measurements. Eur J Orthod 2015 Feb;37(1):13-21.

21. Ladeira DBS, Cruz AD, Almeida SM. Digital panoramic radiography for diagnosis of the temporomandibular joint: CBCT as the gold standard. Braz Oral Res 2015;29(1): 1-7.

22. Hussain AM, Packota G, Major PW, Flores-Mir C. Role of different imaging modalities in assessment of temporomandibular joint erosions and osteophytes: a systematic review. Dentomaxillofac Radiol 2008 Feb;37(2):63-71.

23. Wiese M, Hintze H, Svensson P, Wenzel A. Comparison of diagnostic accuracy of film and digital tomograms for assessment of morphological changes in the TMJ. Dentomaxillofac Radiol 2007 Jan;36(1):12-17.

24. Conti ACCF, Freitas MR, Conti PCR. Avaliação da posição condilar e disfunção temporomandibular em pacientes com má oclusão de Classe II submetidos à protrusão mandibular ortopédica. Dental Press Ortodon Ortop Facial, Maringá 2008 mar-abr;13(2):57-69.

25. Merigue LF, Conti ACCF, Oltramari-Navarro PVP, Navarro RL, Almeida MR. Tomographic evaluation of the temporomandibular joint in malocclusion subjects: condylar morphology and position. Braz Oral Res 2016 Mar;30:e17.

26. Ikeda K, Kawamura A. Assessment of optimal condylar position with limited cone-beam computed tomography. Am J Orthod Dentofacial Orthop 2009 Apr;135(4):495-501. 